\title{
Coronal Alignment of Three Different Types of Implants in Kinematically Aligned Total Knee Arthroplasty: A Comparative Study
}

\author{
Yoshinori Soda1 $^{*}$, Mitsuhiro Nakamura1, Nobuo Adachi ${ }^{2}$ \\ ${ }^{1}$ Department of Orthopaedic Surgery, Hiroshima City Hiroshima Citizens Hospital, Hiroshima, Japan \\ ${ }^{2}$ Department of Orthopaedic Surgery, Integrated Health Sciences, Institute of Biomedical and Health Sciences, Hiroshima \\ University, Hiroshima, Japan \\ Email: *ysoda35@ybb.ne.jp, mitsuhiro@m3.dion.ne.jp, nadachi@hiroshima-u.jp
}

How to cite this paper: Soda, Y., Nakamura, M., \& Adach, N. (2021) Coronal Alignment of Three Different Types of Implants in Kinematically Aligned Total Knee Arthroplasty: A Comparative Study. Open Journal of Orthopedics, 11, 183-198.

https://doi.org/10.4236/ojo.2021.116018

Received: May 12, 2021

Accepted: June 25, 2021

Published: June 28, 2021

Copyright () 2021 by author(s) and Scientific Research Publishing Inc. This work is licensed under the Creative Commons Attribution International License (CC BY 4.0).

http://creativecommons.org/licenses/by/4.0/

\begin{abstract}
Background: The number of total knee arthroplasty (TKA) surgeries performed each year is increasing worldwide and mechanical alignment (MA) is currently seen as the gold standard procedure. However, taking neutral alignment as the universal goal may be mistaken. In our hospital, we currently conduct kinematically aligned TKA (KA-TKA). Three different types of implants are used: the cruciate-retaining (CR) type, cruciate-sacrificing (CS) type, or bi-cruciate-retained (BCR) type. We aimed to compare the coronal alignment observed following KA-TKA and MA-TKA and in normal knees, as well as that achieved with different types of implants. Methods: The study comprised 206 knees of Japanese patients who underwent KA-TKA using varying implants in our Hospital between May 2019 and April 2020. Measurements of pre- and postoperative coronal alignment were determined from weight-bearing full-leg standing radiographs. The postoperative results were compared to measurements taken from patients who underwent MA-TKA $(N=96)$ and normal knees $(N=60)$. Results: No significant differences between the KA-TKA group and normal knees were found for the medial proximal tibial angle (MPTA) $\left(-4.2^{\circ} \pm 2.6^{\circ}\right.$ vs $\left.-3.8^{\circ} \pm 2.5^{\circ}\right)$ or joint line orientation angle (JLOA) $\left(0.2^{\circ} \pm 1.9^{\circ}\right.$ vs $\left.0.3^{\circ} \pm 1.4^{\circ}\right)$. However, when MA-TKA was compared to KA-TKA and normal knees, there were significant differences in both the MPTA and JLOA $(p<0.01)$. Furthermore, for the different implant types, MPTA exhibited significantly greater varus alignment when a CS-type was used than with the other two. Conclusions: Here, we demonstrated that following KA-TKA, the articular surface of the tibia exhibited a similar varus alignment as that of normal knees, meaning that the technique reproduces the native knee. Furthermore, KA is patient-specific,
\end{abstract}


and does not have the same failures as MA-TKA. Therefore, we anticipate a paradigm shift from mechanical to kinematic alignment, which may help reduce the dissatisfaction rate of TKA patients.

\section{Keywords}

Total Knee Arthroplasty, Kinematic Alignment, Mechanical Alignment, Calipered Technique

\section{Background}

Total knee arthroplasty (TKA) is the established gold standard surgical treatment for osteoarthritis of the knee, and the number of procedures performed each year is gradually increasing [1]. Computer-assisted surgery (CAS) has been introduced in recent years, using methods such as navigation systems, patient-specific instruments (PSI), and robots to aid accurate implant placement. As yet, many studies have found that CAS use provides no clinical advantage [2] [3] [4] [5]. Therefore, it may be that taking neutral alignment as the universal goal is mistaken.

Most surgeons are aware that in many normal legs, the Mikulicz line does not pass through the center of the knee [6] [7], and therefore feel that this contradicts the goal of neutral alignment in surgery. This may be why the concept of constitutional varus reported by Bellemans et al. [8] has been accepted without resistance.

In our hospital, we currently conduct calipered kinematically aligned TKA (KA-TKA) following the method reported by Howell [9]. Our goal is to respect the soft tissues and replace the articular surfaces of both the femur and the tibia in order to return the joint to its native or pre-arthritic state while reproducing the patient's own joint line. We use one of three different types of implant: the cruciate-retaining (CR) type, cruciate-sacrificing (CS) type, or bi-cruciate-retaining (BCR) type.

The objective of this paper is to both describe our Simple Surgical Technique, double styluses procedure and cutting block adjusting method, and report and compare our measurements of postoperative coronal alignment from weightbearing full-leg standing radiographs of Japanese patients, following surgery for osteoarthritis of the knee. These subjects tend to exhibit more pronounced varus and curvature than do Europeans and North Americans [10] [11]. This report is the first to evaluate the difference in coronal alignment achieved due to the different types of implant in KA-TKA. Also, we discuss our thoughts on KA-TKA compared to MA-TKA.

\section{Methods}

The study subjects were 206 knees (120 right and 86 left) of patients (40 men and 
166 women of mean age 76 years) who underwent calipered KA-TKA in Hiroshima City Hiroshima Citizens Hospital between May 2019 and April 2020. KA-TKA was performed on all cases except for extra-articular deformity after fracture. The CR-type implants were used in 121 knees, CS-type implants in 49, and BCR-type implants in 36 (Table 1).

Weight-bearing full-leg standing radiographs were scanned preoperatively and 3 weeks after surgery. Patients were instructed to stand with their feet 10 $15 \mathrm{~cm}$ apart with their knees pointing forward. As shown in Figure 1, the preoperative state was assessed by measuring the medial proximal tibial angle (MPTA) and the hip-knee-ankle angle (HKA). The MPTA is defined as the angle of inclination of the tibial axis with respect to the medial articular surface of the tibia (with a negative value denoting varus alignment), and the HKA is defined as the angle between a line joining the center of the femoral head and the distal femoral sulcus, and the tibial axis (with a negative value denoting varus alignment). The postoperative state was evaluated by measuring the MPTA, the joint line orientation angle (JLOA), defined as the angle of inclination of the articular surface of the tibia with respect to the floor (with a negative value denoting an inclination outward and downward), and the HKA.

Table 1. Implant models of KA-TKA $(\mathrm{n}=206)$.

\begin{tabular}{|c|c|c|c|}
\hline Type & Model & Maker & Number \\
\hline \multirow[t]{6}{*}{ CR-type } & Persona CR & (Zimmer Biomet ${ }^{\otimes}$, Warsaw, IN, USA) & 9 \\
\hline & Vanguard ID & (Zimmer Biomet ${ }^{\oplus}$, Warsaw, IN, USA) & 35 \\
\hline & GMK Sphere CR & (Medacta ${ }^{\oplus}$, Castel San Pietro, Switzerland) & 19 \\
\hline & Triathlon CR & (Stryker ${ }^{\circledast}$, Mahwah, NJ, USA) & 43 \\
\hline & BKS TriMax CR & (Ortho Development ${ }^{\oplus}$, Draper, UT, USA) & 15 \\
\hline & & Total & 121 \\
\hline CS-type & GMK Sphere CS & (Medacta ${ }^{\oplus}$, Castel San Pietro, Switzerland) & 49 \\
\hline BCR-type & Vanguard XP & (Zimmer Biomet ${ }^{\oplus}$, Warsaw, IN, USA) & 36 \\
\hline
\end{tabular}

CR, Cruciate retaining; CS, Cruciate sacrificing; BCR, Bi-cruciate retaining; KA, Kinematic alignment.

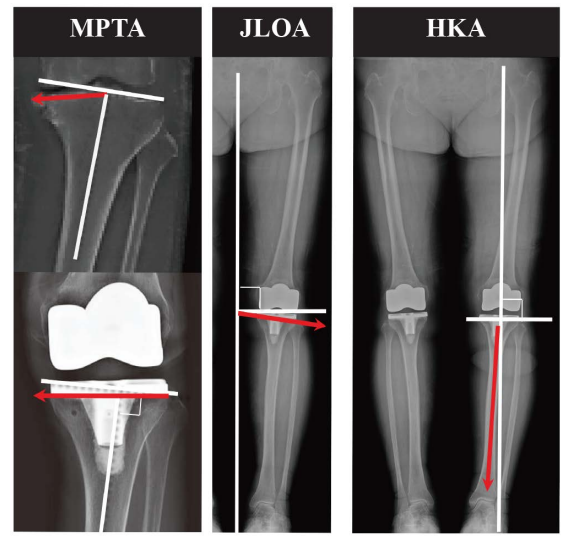

Figure 1. Measurements of coronal alignment from weight-bearing full-leg standing radiographs of Japanese patients. MPTA, medial proximal tibial angle; JLOA, joint line orientation angle; HKA, hip knee ankle angle. 
Furthermore, 96 patients (17 men and 79 women of mean age 76 years) underwent mechanically aligned TKA (MA-TKA) in our hospital (68 CR-type, 10 CS-type, and 18 BCR-type) (Table 2), and the control group comprised 60 normal knees of patients with a ruptured anterior cruciate ligament (ACL) in the opposite knee ( 30 men and 30 women of mean age 26 years). We measured the MPTA, JLOA, and HKA of both groups, and compared their results with those of the KA-TKA group.

Statistical analysis was performed with unpaired Student's t-test using StatView software version 5.0 (SAS Institute, Cary, NC, USA), and $p<0.05$ was considered statistically significant.

All study participants provided written informed consent, and this study was approved by the ethics committee of Hiroshima City Hiroshima Citizens Hospital (no. 2019-199).

\section{Surgical Procedures}

Firstly, the joint is exposed via a medial parapatellar approach. Any damaged cartilage is completely removed from both the distal femur and the posterior condyle, and a referencing guide to compensate for a $2-\mathrm{mm}$ cartilage defect is set against the distal femur (Figure 2). An osteotomy of the same thickness as the distal and posterior parts of the component is performed. The thickness of the resected osteochondral fragment and the kerf of the bone saw are measured together, and the values are recorded on a data sheet. The remains of the meniscus and any osteophytes (particularly on the posterior condyle) are carefully removed.

Table 2. Implant models of MA-TKA $(n=96)$.

\begin{tabular}{|c|c|c|c|}
\hline Type & Model & Maker & Number \\
\hline \multirow[t]{4}{*}{ CR-type } & Vanguard ID & (Zimmer Biomet ${ }^{\oplus}$, Warsaw, IN, USA) & 38 \\
\hline & Triathlon CR & (Stryker ${ }^{\oplus}$, Mahwah, NJ, USA) & 22 \\
\hline & BKS TriMax CR & (Ortho Development ${ }^{\oplus}$, Draper, UT, USA) & 8 \\
\hline & & Total & 68 \\
\hline CS-type & GMK Sphere CS & (Medacta ${ }^{\oplus}$, Castel San Pietro, Switzerland) & 10 \\
\hline BCR-type & Vanguard XP & (Zimmer Biomet $^{\star}$, Warsaw, IN, USA) & 18 \\
\hline
\end{tabular}

CR, Cruciate retaining; CS, Cruciate sacrificing; BCR, Bi-cruciate retaining; MA, Mechanical alignment.
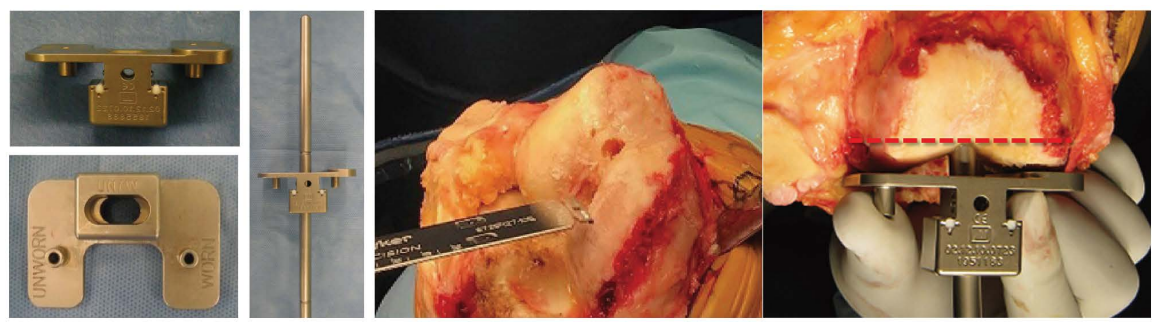

Figure 2. A referencing guide to compensate for a 2-mm cartilage defect is set against the distal femur after removing residual cartilage. 
Next, a trial femoral component is fitted, the ankle is grasped in one hand, and the leg is manually drawn in the distal direction. While the leg is in traction, the distraction gap where the component protrudes furthest is measured; in general, this is in the medial joint in the case of varus deformity, and in the lateral joint in the case of valgus deformity. Because the proximal tibial osteotomy runs parallel to the distal femur, the distraction gap is the difference between the heights of the medial and lateral osteotomies.

For MA-TKA, in the case of varus knee deformity, the tibial axis can usually be measured by placing a tibial osteotomy guide from the lateral intercondylar eminence of the tibia pointing toward the center of the ankle. However, since KA-TKA can be completed in most patients with minimal adjustment, the tibial axis is initially set as a line joining the medial intercondylar eminence of the tibia and the distal tibiofibular joint.

The existence of various types of tibial osteotomy guides means that experience and adjustment are both required when fitting these. When using a cutting block fitted with only a single stylus, this stylus must be removed and adjusted several times, making the procedure more time-consuming. Types that can be fitted with a double variable stylus offer ease of use (Figure 3). The amounts of the medial and lateral osteotomy are determined simultaneously using the stylus, and the cutting block is secured with a pin. Its position is reviewed if it makes varus alignment more pronounced than the axis that was initially set (around $5^{\circ}-6^{\circ}$ varus to the tibial axis). As some patients naturally have a more pronounced medial inclination, this can never be more than a reference axis.

When the tibial surface is uneven or the tibial slope is steep, the surgeon always cannot set the stylus correctly. In such cases, the double styluses procedure is difficult to do; Cutting Block Adjusting Method, as mentioned later, had better be employed. After tibial osteotomy, height is determined with a single stylus and tibial posterior slope is confirmed with "angel wing", after the lateral side of the cutting block is secured with a pin (Figure 4(a)), the tibial shaft alignment

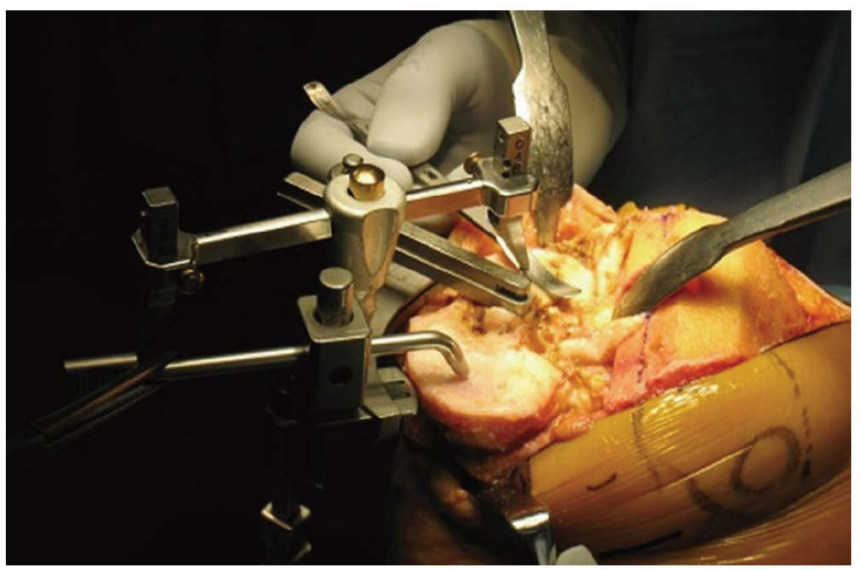

Figure 3. Osteotomy height is determined by setting the double variable styluses on both the medial and lateral tibial articular surface. 


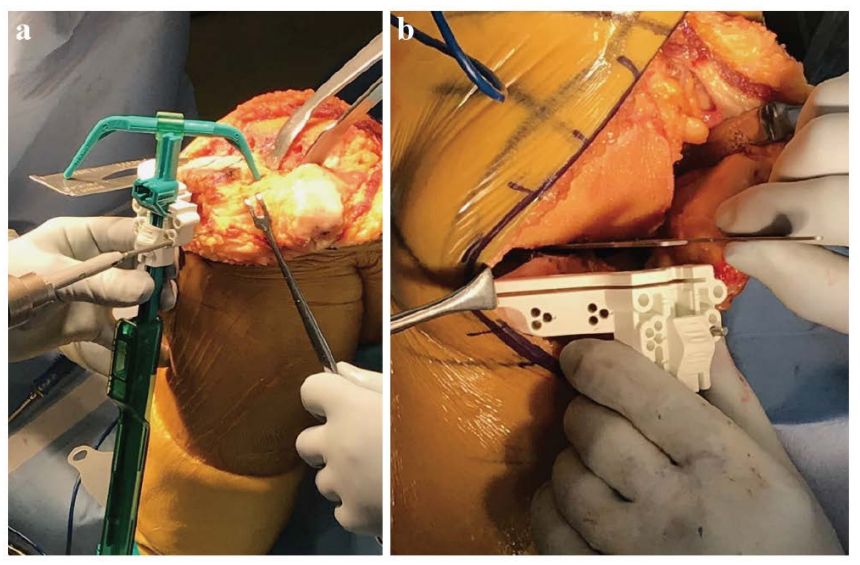

Figure 4. (a) The tibial cutting block is set by first securing with a pin. (b) The cutting block is adjusted parallel to the distal femoral cut surface under distraction of the leg in the knee extended position.

rod is removed. The osteotomy line can subsequently be adjusted, as required, by rotating the cutting block around the axis of the pin while it remains in position (Figure 4(b)). The leg is manually drawn in the distal direction, the pin is secured so that the slot in the cutting block is parallel to the cut surface of the distal femur, and the osteotomy height is reviewed using the stylus. The tibial shaft alignment rod is refitted to stabilize the cutting block during the osteotomy. In this case, too, its position is reviewed if the varus alignment is made more pronounced than the axis that was initially set.

If the posterior cruciate ligament (PCL) is removed (CS-type), the posterior inclination is made somewhat smaller (around $3^{\circ}-5^{\circ}$ ) than the native slope, whereas if the PCL is spared (CR and BCR-types), it should be similar to the native slope, and in principle, the patella is not replaced.

\section{Results}

Socio-demographic characteristics of this study are summarized in Table 3.

In the patients who underwent KA-TKA $(n=206)$, preoperatively the MPTA was $-11.1^{\circ} \pm 7.0^{\circ}$ and the HKA was $-9.9^{\circ} \pm 7.1^{\circ}$, respectively. Postoperatively, the MPTA was $-4.2^{\circ} \pm 2.6^{\circ}$, the JLOA was $0.2^{\circ} \pm 1.9^{\circ}$, and the HKA was $-2.7^{\circ} \pm$ $3.5^{\circ}$, respectively.

In the patients who underwent MA-TKA $(n=96)$, postoperatively the MPTA was $-0.3^{\circ} \pm 1.2^{\circ}$, the JLOA was $-2.2^{\circ} \pm 1.7^{\circ}$, and the HKA was $-0.4^{\circ} \pm 2.3^{\circ}$, respectively. The control group $(n=60)$ displayed an MPTA of $-3.8^{\circ} \pm 2.5^{\circ}$, JLOA of $0.3^{\circ} \pm 1.4^{\circ}$, and an HKA of $-1.2^{\circ} \pm 2.4^{\circ}$, respectively.

A comparison of alignment in knees after KA-TKA and in the control group found that there were no significant differences in the MPTA or JLOA $(p=0.24$ and $p=0.54$, respectively), and their distributions were also equivalent. However, when alignment in the KA-TKA group was compared to that of the MA-TKA group, there were significant differences in both the MPTA and the JLOA $(p<$ 
0.01 ), and their distributions were also clearly different (Figure 5 and Figure 6). The HKA of knees aligned by KA-TKA differed significantly from that of both knees aligned by MA-TKA $(p<0.01)$ and the control group $(p<0.01)$. The HKA of knees aligned by KA-TKA was within $\pm 3^{\circ}$ in only $57 \%$ of patients $(117 / 206)$.

Table 3. Socio-demographic characteristics of this study.

\begin{tabular}{cccc}
\hline & $\mathrm{N}$ & Sex & Age \\
\hline KA-TKA & 206 & $\mathrm{M}: \mathrm{F}=40: 166$ & $76(55-94)$ \\
MA-TKA & 96 & $\mathrm{M}: \mathrm{F}=17: 79$ & $76(50-94)$ \\
Control & 60 & $\mathrm{M}: \mathrm{F}=30: 30$ & $26(13-55)$ \\
\hline
\end{tabular}

KA, Kinematic alignment; MA, Mechanical alignment; Control, Normal knees.

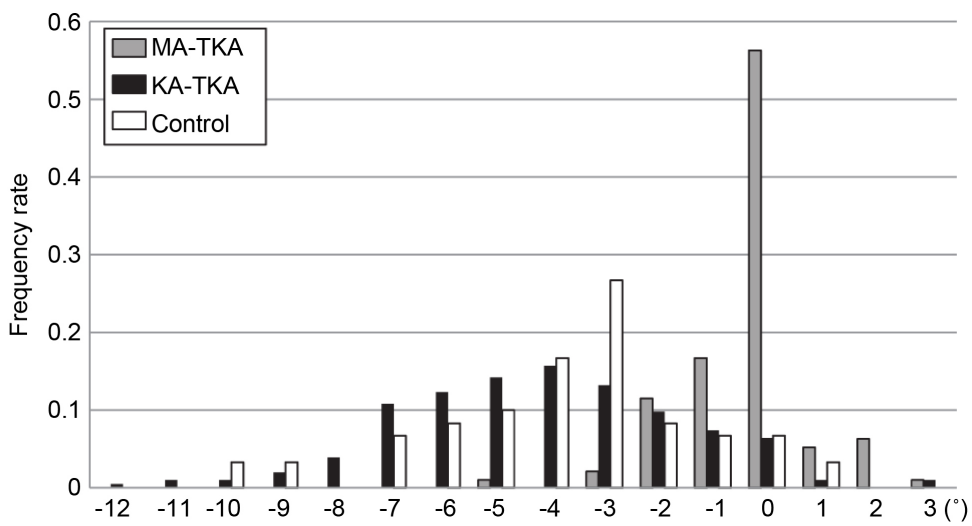

Figure 5. The distribution of MPTA in normal knees and knees following MA-TKA or KA-TKA. The graph shows that KA-TKA and Control group have a normal distribution centered on -3 to -4 degrees, while MA-TKA has a normal distribution centered on 0 degrees. MPTA, medial proximal tibial angle; MA-TKA, mechanically aligned total knee arthroplasty; KA-TKA, kinematically aligned total knee arthroplasty.

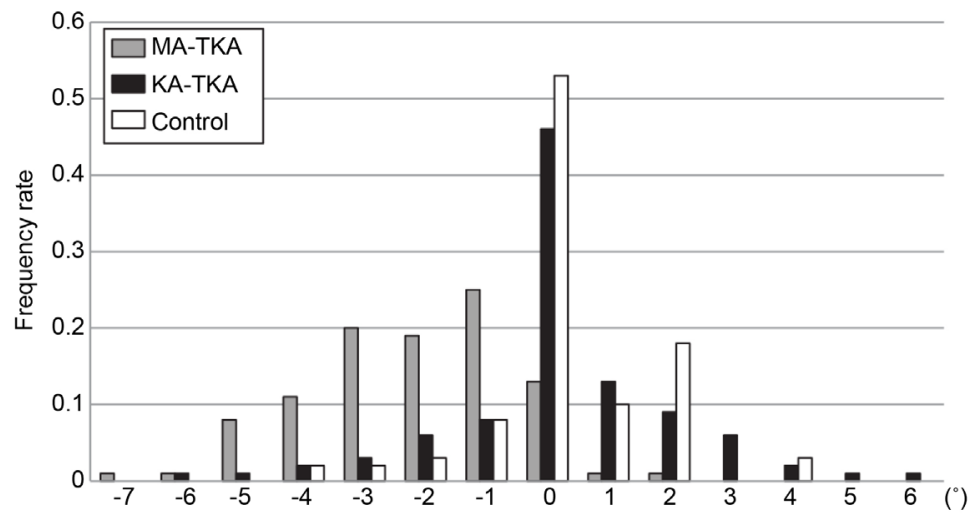

Figure 6. The distribution of JLOA in normal knees and knees following MA-TKA or KA-TKA. The graph shows that KA-TKA and the Control group have a normal distribution centered on 0 degrees, while MA-TKA has a normal distribution centered on -3 to -1 degrees. JLOA, joint line orientation angle; MA-TKA, mechanically aligned total knee arthroplasty; KA-TKA, kinematically aligned total knee arthroplasty. 
Data on the different implants used in KA-TKA are shown in Table 4. There was no difference in either the JLOA or the HKA between different implants, but the MPTA exhibited significantly greater varus alignment when a CS-type implant was used than with either a CR-type $(p<0.05)$ or a BCR-type $(p<0.01)$.

For knees aligned by MA-TKA, there was no difference in any of the parameters between the different implants (Table 5).

Table 4. Radiographic measurements of KA-TKA.

\begin{tabular}{|c|c|c|c|c|c|}
\hline & \multicolumn{2}{|c|}{ Pre-op. } & \multicolumn{3}{|c|}{ Post-op. } \\
\hline & MPTA & HKA & MPTA & JLOA & HKA \\
\hline $\mathrm{CR}(\mathrm{N}=121)$ & $-11.5 \pm 8.0$ & $-9.6 \pm 8.2$ & $-4.1 \pm 2.7^{\S}$ & $0.2 \pm 2.1$ & $-2.4 \pm 3.6$ \\
\hline $\mathrm{CS}(\mathrm{N}=49)$ & $-11.7 \pm 5.7$ & $-11.8 \pm 5.4$ & $-4.9 \pm 2.4^{\S^{*}}$ & $0.3 \pm 1.9$ & $-3.4 \pm 3.1$ \\
\hline $\mathrm{BCR}(\mathrm{N}=36)$ & $-8.2 \pm 4.4$ & $-8.7 \pm 4.5$ & $-3.8 \pm 2.2^{*}$ & $0.0 \pm 1.5$ & $-2.5 \pm 3.4$ \\
\hline Total $(\mathrm{N}=206)$ & $-11.1 \pm 7.0$ & $-9.9 \pm 7.1$ & $-4.2 \pm 2.6$ & $0.2 \pm 1.9$ & $-2.7 \pm 3.5$ \\
\hline
\end{tabular}

Table 5. Post-operative radiographic measurements of MA-TKA.

\begin{tabular}{cccccc}
\hline & \multicolumn{3}{c}{ Pre-op. } & \multicolumn{3}{c}{ Post-op. } \\
\cline { 2 - 6 } & MPTA & HKA & MPTA & JLOA & HKA \\
\hline CR $(\mathrm{N}=68)$ & $-10.6 \pm 7.4$ & $-9.2 \pm 8.5$ & $-0.3 \pm 1.3$ & $-2.1 \pm 1.7$ & $-0.4 \pm 2.3$ \\
CS $(\mathrm{N}=10)$ & $-13.4 \pm 7.1$ & $-13.5 \pm 5.8$ & $-0.2 \pm 1.0$ & $-2.1 \pm 1.5$ & $0.3 \pm 2.5$ \\
BCR $(\mathrm{N}=18)$ & $-8.4 \pm 5.2$ & $-8.6 \pm 4.5$ & $-0.4 \pm 0.8$ & $-2.7 \pm 1.6$ & $0.9 \pm 1.8$ \\
Total $(\mathrm{N}=96)$ & $-10.5 \pm 7.0$ & $-9.5 \pm 7.5$ & $-0.3 \pm 1.2$ & $-2.2 \pm 1.7$ & $-0.4 \pm 2.3$ \\
\hline
\end{tabular}

$\mathrm{CR}$, Cruciate retaining; CS, Cruciate sacrificing; $\mathrm{BCR}, \mathrm{Bi}$-cruciate retaining; MA, Mechanical alignment; MPTA, medial proximal tibial angle; JLOA, joint line orientation angle; HKA, hip knee ankle angle. Valgus measurements are positive, varus measurements are negative.

\section{Discussion}

Here, we demonstrated that following KA-TKA, the articular surface of the tibia exhibited a similar varus alignment as that of normal knees, meaning that the technique reproduces the native knee.

Total knee arthroplasty is an established surgical procedure for the treatment of advanced osteoarthritis that provides good long-term results. This technique boasts a 10 -year postoperative survival rate exceeding $95 \%$ due to improvements in implant durability and design [12] [13]. Mechanical alignment is currently the gold standard procedure, focusing on the accuracy with which the implant can be placed. Although the use of CAS (such as navigation systems, PSI, or robots) may have improved the accuracy of implant placement [14] [15] [16], the clinical outcomes are no different from those of conventional methods that do not involve any extra costs, ironically [1] [2] [3] [4]. The use of these systems thus entails the waste of large amounts of money as well as time. Despite improve- 
ments to the accuracy of implant placement, around $20 \%$ of patients remain dissatisfied [17] [18] [19]. This may be because our current goal of neutral alignment is actually mistaken. Unless we break down the current situation, there may be no prospect of improving the outcomes of TKA 10 or even 20 years into the future.

Mechanical alignment was initially proposed by Freeman et al. [20] and Insall et al. [21]. In the knees in which the PCL is removed, an osteotomy is performed perpendicular to the line from the center of the femoral head to the center of the knee joint. In the lower leg, an osteotomy is performed perpendicular to the functional axis of the tibia, the soft tissues are denuded, and the alignment is finalized, with an under-correction of around $3^{\circ}$ generally considered acceptable. To obtain the same rectangular gap [22], rotation of the femoral component is required. However, if it is rotated too far, reference must be made to anatomical landmarks. Although the context is understandable, the limitations and disadvantages of MA-TKA are now becoming exposed.

The goal of anatomical alignment, which was proposed by Hungerford \& Krackow [23], is the maintenance of the joint line ("joint line theory"). However, this procedure involves a systematic $3^{\circ}$ varus osteotomy of the tibia, and has been discontinued mainly due to issues with the durability of the polyethylene used at the time [24]. In addition, although in Westerners, the mean inclination of the tibial articular surface is $3^{\circ}$ varus, data on healthy Japanese individuals shows that the mean value in this population is $3.8^{\circ}$, and the standard deviation is $2.5^{\circ}$. Therefore, it is dangerous to give all patients in this group a uniform varus inclination of $3^{\circ}$; rather, osteotomy must be patient-specific. This is a limitation of both systemic alignment and anatomical alignment.

Although numerous studies have reported that obtaining neutral alignment results in good clinical outcomes [25] [26] [27] [28] [29], an even larger number have found that this is not the case [30]-[35]. However, as described by Rivière [36], kinematic alignment is patient-specific, and does not have the same failures as an anatomical alignment or MA-TKA, which involve systematic osteotomies.

Our results that after KA-TKA, the articular surface of the tibia exhibited a similar varus alignment with respect to the tibial axis as that of normal knees and was parallel to the floor are consistent with previous reports [37] [38]. The difference in the HKA between the KA-TKA group and the control was considered to be due to the age difference between the two groups (mean age 76 years and 26 years, respectively). Therefore, our KA-TKA technique reproduces the native \& pre-arthritic knee condition.

The data on the comparison of JLOA in knees aligned by KA-TKA or MA-TKA and normal knees were almost entirely consistent with those reported by Ji et al. [38]. Although static and dynamic data are not necessarily associated [39], when the articular surface of the tibia is parallel with the floor in the same way as in normal knees, knee adduction movement (KAM) is smaller, as reported by Niki et al. [40]. This is believed to alleviate shear force during weight-bearing. Other studies have found that KA-TKA provides better gait 
function than does MA-TKA [41], and may contribute to improving not only the survival rate but also patient satisfaction.

Recent studies have also compared the short-term clinical outcomes of KA and MA-TKA, none of which have concluded that KA-TKA is inferior [42] [43] [44]. Although concerns about survivorship are one reason that many surgeons remain skeptical about KA-TKA, good 10-year postoperative outcomes have been reported by Howell et al. [45]. Further research should be done in the future to clarify these results.

Since osteotomy in MA-TKA is systematic, it produces no differences in alignment between different implants. However, following KA-TKA, the inclination of the tibial articular surface exhibited greater varus alignment when a CS-type implant was used compared with the other two types of implant, an extremely interesting result. We conjecture that this was because the procedure we used is a soft-tissue-respecting surgical technique that is dependent on the femoral component. Furthermore, we speculate that removing both cruciate ligaments causes the extension alignment under traction to become slightly more varus. Accordingly, when an implant that requires the resection of both cruciate ligaments is used, the tibial component is placed in an excessively varus alignment and may cause mechanical strain [46] [47]. Therefore, it may be better to combine the implant with a design such as a ball-in-socket shape or to use a CR-type or BCR-type implant, which preserves the posterior cruciate ligament.

Consideration must also be given to disorders for which KA-TKA is not indicated [48]. One of the guiding principles of KA is that it is a soft-tissue-respecting surgery. Contraindications thus include: 1) patients with contracture that cannot be corrected even after bone spur resection, 2) patients with joint instability due to soft tissue breakdown, and 3) patients with extra-articular deformity. However, as even severe deformity can be corrected by osteophyte removal in some patients, in principle, the decision on whether to perform KA-TKA is made intraoperatively. Another contraindication considered for KA-TKA is the difficult-to-correct fixed valgus knee, as it is not feasible to respect the soft tissue, although this condition is rare. However, it may still be necessary to reconsider the merits and demerits of aiming for neutral alignment to the point of unnecessarily destroying soft tissue in these patients. For the choice of an implant in these conditions, the best is the BCR-type or unicompartmental knee arthroplasty (UKA), which preserves the soft tissues involved in controlling knee movements (particularly the ACL and PCL). Given that the rotational axis of the knee is the medial joint, an implant that restricts the medial joint in the coronal section, such as a CR-type, is preferable. However, a medial pivot implant type that restricts the medial joint in both the coronal and sagittal sections, such as a posterior stabilizing (PS)-type, is favored over an implant with a post-cam structure in the center of the knee. Further studies are required to identify differences in outcomes when different types of implants are used.

A final consideration may be that we are being deceived by postoperative 
frontal X-rays after TKA. We have always thought that correct frontal X-rays of the knee are those in which the tibial axis is parallel to the vertical axis and the articular surface is parallel to the horizontal axis on short films. However, given that human frontal X-rays are scanned in line with the anatomical position, it is clear that the axis of the lower leg cannot run parallel to the vertical axis. Therefore, frontal knee X-rays should be scanned with the body in an inclined state.

The current study presented various limitations. Firstly, we assessed the validity of the surgical procedures purely in terms of X-ray measurements and included no descriptions of clinical outcomes. Secondly, the method and timing for capturing the standing X-rays of both legs were not strictly determined or applied for all patients. Finally, we carried out only a static assessment of the effect of the surgical procedure without indicating the association with dynamic assessment. Future studies should address these limitations to obtain a complete understanding of KA-TKA.

\section{Conclusion}

We are by no means decrying mechanical alignment, which has been the golden standard for many years. However, this paper has demonstrated that it may be possible to reduce the $20 \%$ dissatisfaction rate of TKA patients by fully grasping the concept of kinematic alignment and reproducing the joint lines of individual patients while maintaining the concepts of respecting soft tissue and resurfacing surgery. Since KA-TKA is a soft tissue respecting the procedure, the preserved ligaments (particularly the ACL and PCL) affect component placement. Therefore, it is important to remember that the placement of the tibial component may vary depending on the type of implant used. We anticipate that in the near future, there will be a paradigm shift in TKA from mechanical alignment, which systematically aims for neutral alignment, to kinematic alignment, which involves patient-specific, personalized alignment.

\section{Acknowledgements}

Special thanks to Editage for their assistance with English-language editing for this manuscript.

\section{Conflicts of Interest}

All authors have completed the ICMJE uniform disclosure form. The authors have no conflicts of interest to declare.

\section{Authors' Contributions}

1) Conception and design: Yoshinori Soda.

2) Administrative support: Yoshinori Soda.

3) Provision of study materials or patients: Yoshinori Soda\& Mitsuhiro Nakamura.

4) Collection and assembly of data: Yoshinori Soda.

5) Data analysis and interpretation: All authors. 
6) Manuscript writing: All authors.

7) Final approval of manuscript: All authors.

\section{Availability of Data and Materials}

The datasets used and/or analyzed during the current study are available from the corresponding author on reasonable request.

\section{Reporting Checklist Statement}

The authors have completed the STROBE reporting checklist.

\section{Ethical Statement}

The authors are accountable for all aspects of the work in ensuring that questions related to the accuracy or integrity of any part of the work are appropriately investigated and resolved. The study was conducted in accordance with the Declaration of Helsinki (as revised in 2013). The study was approved by the ethics committee of Hiroshima City Hiroshima Citizens Hospital (No. 2019-199) and written informed consent was taken from all individual participants.

\section{References}

[1] Singh, J.A., Yu, S., Chen, L. and Cleveland, J.D. (2019) Rates of Total Joint Replacement in the United States: Future Projections to 2020-2040 Using the National Inpatient Sample. The Journal of Rheumatology, 46, 1134-1140. https://doi.org/10.3899/jrheum.170990

[2] Lee, D.Y., Park, Y.J., Hwang, S.C., Park, J.S. and Kang, D.G. (2020) No Differences in Mid- to Long-Term Outcomes of Computer-Assisted Navigation versus Conventional Total Knee Arthroplasty. Knee Surgery, Sports Traumatology, Arthroscopy, 28, 3183-3192. https://doi.org/10.1007/s00167-019-05808-5

[3] Kim, Y.H., Park, J.W. and Kim, J.S. (2018) 2017 Chitranjan S. Ranawat Award: Does Computer Navigation in Knee Arthroplasty Improve Functional Outcomes in Young Patients? A Randomized Study. Clinical Orthopaedics and Related Research, 476, 6-15. https://doi.org/10.1007/s11999.0000000000000000

[4] Ollivier, M., Parratte, S., Lino, L., Flecher, X., Pesenti, S. and Argenson, J.N. (2018) No Benefit of Computer-Assisted TKA: 10-Year Results of a Prospective Randomized Study. Clinical Orthopaedics and Related Research, 476, 126-134. https://doi.org/10.1007/s11999.0000000000000021

[5] Antonios, J.K., Kang, H.P., Robertson, D., Oakes, D.A., Lieberman, J.R. and Heckmann, N.D. (2020) Population-Based Survivorship of Computer-Navigated versus Conventional Total Knee Arthroplasty. The Journal of the American Academy of Orthopaedic Surgeons, 28, 857-864. https://doi.org/10.5435/JAAOS-D-19-00548

[6] Tang, W.M., Zhu, Y.H. and Chiu, K.Y. (2000) Axial Alignment of the Lower Extremity in Chinese Adults. The Journal of Bone and Joint Surgery. American Volume, 82, 1603-1608. https://doi.org/10.2106/00004623-200011000-00014

[7] Jabalameli, M., Moghimi, J., Yeganeh, A. and Nojomi, M. (2015) Parameters of Lower Extremities Alignment View in Iranian Adult Population. Acta Medica Iranica, 53, 293-296.

[8] Bellemans, J., Colyn, W., Vandenneucker, H. and Victor, J. (2012) The Chitranjan 
Ranawat Award: Is Neutral Mechanical Alignment Normal for All Patients? The Concept of Constitutional Varus. Clinical Orthopaedics and Related Research, 470, 45-53. https://doi.org/10.1007/s11999-011-1936-5

[9] Howell, S.M. (2019) Calipered Kinematically Aligned Total Knee Arthroplasty: An Accurate Technique That Improves Patient Outcomes and Implant Survival. Orthopedics, 42, 126-135. https://doi.org/10.3928/01477447-20190424-02

[10] Kim, Y.H., Matsuda, S. and Kim, T.K. (2016) Clinical Faceoff: Do We Need Special Strategies for Asian Patients with TKA? Clinical Orthopaedics and Related Research, 474, 1102-1107. https://doi.org/10.1007/s11999-016-4716-4

[11] Kawasaki, M., Nagamine, R., Chen, W., Ma, Y., Sakai, A. and Suguro, T. (2020) Proximal Tibia Vara Involves the Medial Shift of the Tibial Articular Surface. Journal of Orthopaedic Surgery (Hong Kong), 28, 1-5.

https://doi.org/10.1177/2309499020902592

[12] Knutson, K. and Robertsson, O. (2010) The Swedish Knee Arthroplasty Register (www.knee.se). Acta Orthopaedica, 81, 5-7. https://doi.org/10.3109/17453671003667267

[13] Paxton, E.W., Furnes, O., Namba, R.S., Inacio, M.C., Fenstad, A.M. and Havelin, L.I. (2011) Comparison of the Norwegian Knee Arthroplasty Register and a United States Arthroplasty Registry. The Journal of Bone and Joint Surgery. American Volume, 93, 20-30. https://doi.org/10.2106/JBJS.K.01045

[14] Matziolis, G., Krocker, D., Weiss, U., Tohtz, S. and Perka, C. (2007) A Prospective, Randomized Study of Computer-Assisted and Conventional Total Knee Arthroplasty. Three-Dimensional Evaluation of Implant Alignment and Rotation. The Journal of Bone and Joint Surgery. American Volume, 89, 236-243. https://doi.org/10.2106/JBJS.F.00386

[15] Liow, M.H.L., Xia, Z. and Wong, M.K. (2004) Robot-Assisted Total Knee Arthroplasty Accurately Restores the Joint Line and Mechanical Axis. A Prospective Randomized Study. The Journal of Arthroplasty, 29, 2373-2377. https://doi.org/10.1016/j.arth.2013.12.010

[16] Decking, R., Markmann, M., Fuchs, J., Puhl, W. and Scharf, H.P. (2005) Leg Axis after Computer-Navigated Total Knee Arthroplasty: A Prospective Randomized Trial Comparing Computer-Navigated and Manual Implantation. The Journal of Arthroplasty, 20, 282-288. https://doi.org/10.1016/j.arth.2004.09.047

[17] Bourne, R.B., Chesworth, B.M., Davis, A.M., Mahomed, N.N. and Charron, K.D. (2010) Patient Satisfaction after Total Knee Arthroplasty: Who Is Satisfied and Who Is Not? Clinical Orthopaedics and Related Research, 468, 57-63. https://doi.org/10.1007/s11999-009-1119-9

[18] Nam, D., Nunley, R.M. and Barrack, R.L. (2014) Patient Dissatisfaction Following Total Knee Replacement: A Growing Concern? The Bone \& Joint Journal, 96, 96-100. https://doi.org/10.1302/0301-620X.96B11.34152

[19] Baker, P.N., van der Meulen, J.H., Lewsey, J. and Gregg, P.J. (2007) The Role of Pain and Function in Determining Patient Satisfaction after Total knee Replacement. Data from the National Joint Registry for England and Wales. The Journal of Bone and Joint Surgery. British Volume, 89, 893-900. https://doi.org/10.1302/0301-620X.89B7.19091

[20] Freeman, M.A., Swanson, S.A. and Todd, R.C. (1973) Total Replacement of the Knee Using the Freeman-Swanson Knee Prosthesis. Clinical Orthopaedics and Related Research, 94, 153-170. https://doi.org/10.1097/00003086-197307000-00020

[21] Insall, J.N., Binazzi, R., Soudry, M. and Mestriner, L.A. (1985) Total Knee Arthrop- 
lasty. Clinical Orthopaedics and Related Research, 192, 13-22. https://doi.org/10.1097/00003086-198501000-00003

[22] Griffin, F.M., Insall, J.N. and Scuderi, G.R. (2000) Accuracy of Soft Tissue Balancing in Total Knee Arthroplasty. The Journal of Arthroplasty, 15, 970-973. https://doi.org/10.1054/arth.2000.6503

[23] Hungerford, D.S. and Krackow, K.A. (1985) Total Joint Arthroplasty of the Knee. Clinical Orthopaedics and Related Research, 192, 23-33. https://doi.org/10.1097/00003086-198501000-00004

[24] Rivière, C., Lazic, S., Boughton, O., Wiart, Y., Villet, L. and Cobb, J. (2018) Current Concepts for Aligning Knee Implants: Patient-Specific or Systematic? EFORT Open Reviews, 3, 1-6. https://doi.org/10.1302/2058-5241.3.170021

[25] Lotke, P.A. and Ecker, M.L. (1977) Influence of Positioning of Prosthesis in Total Knee Replacement. The Journal of Bone and Joint Surgery. American Volume, 59, 77-79. https://doi.org/10.2106/00004623-197759010-00013

[26] Tew, M. and Waugh, W. (1985) Tibiofemoral Alignment and the Results of Knee Replacement. The Journal of Bone and Joint Surgery. British Volume, 67, 551-556. https://doi.org/10.1302/0301-620X.67B4.4030849

[27] Fang, D.M., Ritter, M.A. and Davis, K.E. (2009) Coronal Alignment in Total Knee Arthroplasty: Just How Important Is It? The Journal of Arthroplasty, 24, 39-43. https://doi.org/10.1016/j.arth.2009.04.034

[28] Ritter, M.A., Davis, K.E., Meding, J.B., Pierson, J.L., Berend, M.E. and Malinzak, R.A. (2011) The Effect of Alignment and BMI on Failure of Total Knee Replacement. The Journal of Bone and Joint Surgery. American Volume, 93, 1588-1596. https://doi.org/10.2106/JBJS.J.00772

[29] Jeffery, R.S., Morris, R.W. and Denham, R.A. (1991) Coronal Alignment after Total Knee Replacement. The Journal of Bone and Joint Surgery. British Volume, 73, 709-714. https://doi.org/10.1302/0301-620X.73B5.1894655

[30] Bonner, T.J., Eardley, W.G., Patterson, P. and Gregg, P.J. (2011) The Effect of Post-Operative Mechanical Axis Alignment on the Survival of Primary Total Knee Replacements after a Follow-Up of 15 Years. The Journal of Bone and Joint Surgery. British Volume, 93, 1217-1222. https://doi.org/10.1302/0301-620X.93B9.26573

[31] Parratte, S., Pagnano, M.W., Trousdale, R.T. and Berry, D.J. (2010) Effect of Postoperative Mechanical Axis Alignment on the Fifteen-Year Survival of Modern, Cemented Total Knee Replacements. The Journal of Bone and Joint Surgery. American Volume, 92, 2143-2149. https://doi.org/10.2106/JBJS.I.01398

[32] Lee, S.S., Lee, H., Lee, D.H. and Moon, Y.W. (2018) Slight Under-Correction Following Total Knee Arthroplasty for a Valgus Knee Results in Similar Clinical Outcomes. Archives of Orthopaedic and Trauma Surgery, 138, 1011-1019. https://doi.org/10.1007/s00402-018-2957-2

[33] Magnussen, R.A., Weppe, F., Demey, G., Servien, E. and Lustig, S. (2011) Residual Varus Alignment Does Not Compromise Results of TKAs in Patients with Preoperative Varus. Clinical Orthopaedics and Related Research, 469, 3443-3450. https://doi.org/10.1007/s00402-018-2957-2

[34] Shelton, T.J., Nedopil, A.J., Howell, S.M. and Hull, M.L. (2017) Do Varus or Valgus Outliers Have Higher Forces in the Medial or Lateral Compartments than Those Which Are In-Range after a Kinematically Aligned Total Knee Arthroplasty? Limb and Joint Line Alignment after Kinematically Aligned Total Knee Arthroplasty. The Bone \& Joint Journal, 99, 1319-1328. https://doi.org/10.1302/0301-620X.99B10.BJJ-2017-0066.R1 
[35] Abdel, M.P., Ollivier, M., Parratte, S., Trousdale, R.T., Berry, D.J. and Pagnano, M.W. (2018) Effect of Postoperative Mechanical Axis Alignment on Survival and Functional Outcomes of Modern Total Knee Arthroplasties with Cement: A Concise Follow-Up at 20 Years. The Journal of Bone and Joint Surgery. American Volume, 100, 472-478. https://doi.org/10.2106/JBJS.16.01587

[36] Rivière, C. (2019) Letter to the Chief Editor Regarding a Recently Published Article Entitled "No Difference in Outcomes and Gait Analysis between Mechanical and Kinematic Knee Alignment Methods Using Robotic Total Knee Arthroplasty". Knee Surgery, Sports Traumatology, Arthroscopy, 27, 2040-2041. https://doi.org/10.1007/s00167-019-05545-9

[37] Hutt, J., Massé, V., Lavigne, M. and Vendittoli, P.A. (2016) Functional Joint Line Obliquity after Kinematic Total Knee Arthroplasty. International Orthopaedics, 40, 29-34. https://doi.org/10.1007/s00264-015-2733-7

[38] Ji, H.M., Han, J., Jin, D.S., Seo, H. and Won, Y.Y. (2016) Kinematically Aligned TKA Can Align Knee Joint Line to Horizontal. Knee Surgery, Sports Traumatology, Arthroscopy, 24, 2436-2441. https://doi.org/10.1007/s00167-016-3995-3

[39] Miller, E.J., Pagnano, M.W. and Kaufman, K.R. (2014) Tibiofemoral Alignment in Posterior Stabilized Total Knee Arthroplasty: Staticalignment Does Not Predict Dynamic Tibial Plateau Loading. Journal of Orthopaedic Research, 32, 1068-1074. https://doi.org/10.1002/jor.22644

[40] Niki, Y., Nagura, T., Nagai, K., Kobayashi, S. and Harato, K. (2018) Kinematically Aligned Total Knee Arthroplasty Reduces Knee Adduction Moment More than Mechanically Aligned Total Knee Arthroplasty. Knee Surgery, Sports Traumatology, Arthroscopy, 26, 1629-1635. https://doi.org/10.1007/s00167-017-4788-Z

[41] Blakeney, W., Clément, J., Desmeules, F., Hagemeister, N., Rivière, C. and Vendittoli, P.A. (2019) Kinematic Alignment in Total Knee Arthroplasty Better Reproduces Normal Gait than Mechanical Alignment. Knee Surgery, Sports Traumatology, Arthroscopy, 27, 1410-1417. https://doi.org/10.1007/s00167-017-4788-Z

[42] Courtney, P.M. and Lee, G.C. (2017) Early Outcomes of Kinematic Alignment in Primary Total Knee Arthroplasty: A Meta-Analysis of the Literature. The Journal of Arthroplasty, 32, 2028-2032. https://doi.org/10.1016/j.arth.2017.02.041

[43] Li, Y., Wang, S., Wang, Y. and Yang, M. (2018) A Systematic Review and Meta-Analysis. Journal of Knee Surgery, 31, 78-86. https://doi.org/10.1055/s-0037-1602136

[44] Maderbacher, G., Keshmiri, A., Krieg, B., Greimel, F., Grifka, J. and Baier, C. (2019) Kinematic Component Alignment in Total Knee Arthroplasty Leads to Better Restoration of Natural Tibiofemoral Kinematics Compared to Mechanic Alignment. Knee Surgery, Sports Traumatology, Arthroscopy, 27, 1427-1433. https://doi.org/10.1007/s00167-018-5105-1

[45] Howell, S.M., Shelton, T.J. and Hull, M.L. (2018) Implant Survival and Function Ten Years after Kinematically Aligned Total Knee Arthroplasty. The Journal of Arthroplasty, 33, 3678-3684. https://doi.org/10.1016/j.arth.2018.07.020

[46] Nakamura, S., Tian, Y., Tanaka, Y., Kuriyama, S., Ito, H., Furu, M. and Matsuda, S. (2017) The Effects of Kinematically Aligned Total Knee Arthroplasty on Stress at the Medial Tibia: A Case Study for Varus Knee. Bone \& Joint Research, 6, 43-51. https://doi.org/10.1302/2046-3758.61.BJR-2016-0090.R1

[47] Ishikawa, M., Kuriyama, S., Ito, H., Furu, M., Nakamura, S. and Matsuda, S. (2015) Kinematic Alignment Produces Near-Normal Knee Motion but Increases Contact Stress after Total Knee Arthroplasty: A Case Study on a Single Implant Design. 
Knee, 22, 206-212. https://doi.org/10.1016/j.knee.2015.02.019

[48] Rivière, C., Iranpour, F., Auvinet, E., Howell, S., Vendittoli, P.A., Cobb, J. and Parratte, S. (2017) Alignment Options for Total Knee Arthroplasty: A Systematic Review. Orthopaedics \& Traumatology: Surgery \& Research, 103, 1047-1056. https://doi.org/10.1016/j.otsr.2017.07.010 